\title{
Survival in Transthyretin Familial Amyloid Polyneuropathy: A Review Monica Ines ${ }^{1}$, Joao Costa ${ }^{1,2,3 *}$
}

'Instituto de Medicina Molecular, Faculdade de Medicina, Universidade de Lisboa, Av. Prof. Egas Moniz, 1649-028, Lisboa, Portugal ${ }^{2}$ Laboratório de Farmacologia Clínica e Terapêutica, Faculdade de Medicina, Universidade de Lisboa, Av. Prof. Egas Moniz, 1649-028, Lisboa, Portugal ${ }^{3}$ Centro de Estudos de Medicina Baseada na Evidência, Faculdade de Medicina, Universidade de Lisboa, Av. Prof. Egas Moniz, 1649-028, Lisboa, Portugal

\section{Article Info}

\section{Article Notes}

Received: January 22, 2019

Accepted: February 14, 2019

\section{*Correspondence:}

Dr. João Costa, Faculdade de Medicina, Universidade de Lisboa, Av. Prof. Egas Moniz, 1649-028 Lisboa, Portugal; Telephone No: +351 217940424; Email: jncosta@medicina.ulisboa.pt.

(c) 2019 Costa J. This article is distributed under the terms of the Creative Commons Attribution 4.0 International License

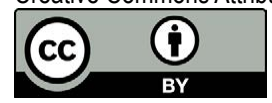

Keywords:

Amyloidosis

Transthyretin Familial Amyloid Polyneuropathy

Liver Transplantation

Tafamidis

Survival
Abstract

Transthyretin-associated familial amyloid polyneuropathy (TTR-FAP) is a neurological disease that affects severely patients and their families and caregivers over generations. It is a rare, progressive, and if untreated fatal autosomal dominant hereditary disorder. The disease may affect multiple organ systems and if untreated progress rapidly to death. TTR-FAP affects nearly 10,000 people worldwide, with known endemic regions in Portugal, Sweden, and Japan. Until recently only liver transplantation and tafamidis were treatment options across several world regions. Despite the worldwide use of these disease-modifying treatments to delay disease progression, challenges in clinical assessment and management remain because of disease heterogeneity, phenotypic diversity, small patient populations, incomplete natural history and uncertainty of treatment effect in survival. The two new treatment options (inotersen and patisiran) appear to provide important benefits for patients, based on clinical trials short-term evidence. In this review, we discuss the disease natural survival course and currently available treatments impact on survival. We also discuss the importance of treatment choice (and or sequence of treatments) to maximize survival, while preserving the patient's health-related quality of life.

\section{Introduction}

Transthyretin-associated familial amyloid polyneuropathy (TTR-FAP) is an autosomal-dominant, adult-onset, progressive neurodegenerative systemic disease, first identified by Corino de Andrade in Portugal ${ }^{1}$. TTRFAP symptoms stem from amyloidosis that results from misfolding of the transthyretin (TTR) protein tetramer, leading to TTR fibrils and other aggregates $^{2-5}$. More than 120 TTR gene mutations have been described, the most common of which associated with TTR-FAP is Val30Met ${ }^{6}$. The disease is rare; it affects 8000 to 10,000 people worldwide, with the endemic group of patients mainly found in Portugal, Sweden, and $\operatorname{Japan}^{7-9}$. Both sexes are affected, the disease onset occurs mainly in young adulthood (median 33 years age) and if untreated, it progresses rapidly to death, usually within 10 years after symptom onset, depending on the endemic region, genotype, symptoms, and other factors ${ }^{2}$. Common causes of death among untreated patients include cachexia, cardiac failure, arrhythmia and secondary infections ${ }^{10,11}$. The disease imposes a substantial burden characterized by high levels of patient's impairment in physical health, quality of life, reduced productivity and a negative impact on the mental health of families and caregivers ${ }^{12}$.

Liver transplantation (LTx) was first implemented in 1990 as a surgical treatment option to halt or ameliorate familial TTR amyloidosis ${ }^{13}$. Because $95 \%$ of plasma TTR is of hepatic origin, LTx substantially reduces the concentration of mutant TTR in the blood ${ }^{14,15}$. Despite the benefits of LTX, improved survival is attained at the expense of long waiting times for 
an organ, lifelong immunosuppressive therapy, significant early mortality, and postoperative complications which can impact long-term health-related quality of life ${ }^{16-19}$. Causes of death after LTx include secondary to septicaemia (22\%), cardiovascular-related deaths (22\%), liver-related complications (14\%) and intraoperative death $(3 \%)^{17}$. Also, in some cases, polyneuropathy has been reported to progress after $\mathrm{LTX}^{20}$.

Diflunisal is a non-steroidal anti-inflammatory drug (NSAID) developed in the 1970s. In Europe, the drug is not generally available and has occasional authorization use on a country basis. A randomized multicentre, placebocontrolled phase 3 study confirmed that diflunisal at a dose of $250 \mathrm{mg}$ twice daily slows the progression of the neuropathy ${ }^{21}$. However, the safety concerns with long-term NSAIDs use may limit its use in real world clinical setting among TTR-FAP patients ${ }^{22}$.

Tafamidis, an oral, potent and selective TTR kinetic stabiliser that slows tetramer dissociation and amyloid genesis became available as a treatment option at $20 \mathrm{mg}$ once daily, first in the context of clinical trials (2007) and later as an approved drug (November 2011 in the European Union) for the treatment of adult TTR-FAP patients in stage 1 to delay disease progression ${ }^{23,24}$. Tafamidis is currently the standard of care ${ }^{25}$; disease progression delay, quality of life preservation, nutritional status improvement and a favourable safety profile in long-term use has been demonstrated $^{26-30}$. Most common adverse effects $(\geq 1 / 10)$ in tafamidis-treated patients include urinary tract infections, vaginal infections, upper abdominal pain, and diarrhoea.

Two new treatment options were recently approved for the treatment of adult patients in stage 1 or stage 2 transthyretin amyloid polyneuropathy disease ${ }^{31,32}$. Inotersen, a 2'-0-methoxyethyl-modified antisense oligonucleotide, inhibits hepatic production of transthyretin. Treatment with subcutaneous inotersen $300 \mathrm{mg}$ once weekly improved the course of neurologic disease and quality of life compared with placebo. Nevertheless, thrombocytopenia and renal dysfunction (including glomerulonephritis) are important safety concerns, managed with enhanced monitoring ${ }^{31}$. Patisiran, an RNA interference therapeutic delivered via intravenous infusion, specifically inhibits hepatic synthesis of transthyretin. This treatment $(0.3 \mathrm{mg}$ per kilogram of body weight, once every 3 weeks) significantly improved multiple clinical manifestations of hereditary transthyretin amyloidosis including significant improvements in neuropathy, quality of life, walking, nutritional status, and activities of daily living as compared with placebo. The most commonly reported adverse events in patisirantreated patients included peripheral edema and infusionrelated reactions. Notably, the polyneuropathy disability score improved in some $(8 \%)$ patients which included the transition from assisted to unassisted walking, an outstanding outcome for patients consistent with the effect of the medicine on gait speed ${ }^{32,33}$.

In the present work, we review the TTR-FAP disease natural course and currently available treatments impact on survival. We also reflect on treatment choice (and sequence of treatments), given the available treatments and the newly approved medicines. The clinical aim is to stabilize/halt disease progression in all possible patients, including those in more advanced stages, hence decreasing the disease excess mortality.

\section{Literature Review}

We searched Medline and Embase (Ovid interface), with the search terms "transthyretin*" OR ("amyloid* AND [family* OR heredit*]) combined with the terms "polyneuropathy" OR "neuropathy*" for the definition of the population (transthyretin-associated familial amyloid polyneuropathy [TTR-FAP]). The terms "natural history", "survival", and "mortality" were used for the definition of the outcomes of interest. To increase sensitivity, search terms were used as both indexed and free-text terms, without any language or type of study restrictions. After deduplication, a total of 455 references were independently screened to identify potential studies characterizing the natural history of TTR-FAP and evaluating the effect of treatment on the natural history (survival) of the disease.

Limited number of studies report the natural history of TTR-FAP and treatment impact, particularly as it relates to survival and its determinants. In the largest cohort study published, 3160 consecutive Portuguese patients with Val30Met were followed up since the first patient presentation ${ }^{34}$. The natural history of the disease was characterized by a median overall survival (mOS) of 12 years, with men and patients with late-onset disease facing significantly worse prognosis and a lower survival, if untreated. The median overall survival in the LTx cohort was 21 years since transplantation (maximum follow-up, 25 years). The median overall survival in the tafamidis cohort has not been reached, as the maximum followup for this cohort was 10 years. In both treated cohorts, the mortality risk was higher among patients with longer disease duration before treatment initiation. The 10-year survival rates for the most recent LTx subgroup (year 2007 and onwards) and tafamidis-treated cohorts were $85 \%$ and $93 \%$, respectively. In comparison with the untreated cohort of patients, both LTx and tafamidis significantly improved survival in stage 1 Val30Met patients with early-onset $<<50$ years) disease, with $75 \%$ and $91 \%$ reductions in mortality risk, respectively. Among this population, tafamidis was associated with significant survival gains compared with LTx, a $63 \%$ reduction in mortality risk. However, nearly $11 \%$ of tafamidis-treated patients have undergone posterior 
LTx. Some authors argue that early-stage patients should be treated initially with tafamidis, and only undergone LTx if their disease progressed while on oral treatment ${ }^{35}$. On the other hand, other authors found this treatment strategy questionable ${ }^{17}$ because the patient prognosis would be worse for liver transplantation. In the Portuguese survival study this treatment sequence strategy was studied and it was found that previous tafamidis treatment did not worsen the survival prognosis for patients who later underwent LTx as compared with LTx treatment only, which can be very relevant for medical treatment sequence decision. For older late-onset patients ( $\geq 50$ years) LTx is not a general treatment option, hence only tafamidis was compared with an untreated cohort. Among patients with late-onset disease tafamidis was associated with a significant $82 \%$ reduction in mortality risk. Early onset of disease, shorter disease duration, and tafamidis treatment were found to be independent favourable and significant factors for survival.

We found two other studies characterizing the natural course of the disease which evaluated the effect of treatment (LTX) on survival. The Sweden survival study included 141 Val30Met patients (LTx, 108; untreated, 33) and has shown that patients who underwent LTx had significantly increased survival rate compared with untreated patient. Untreated patients had a median overall survival (mOS) of 12 years and 10 and 15 year survival rates of $62 \%$ and $19 \%$, respectively. Patients who underwent LTx had 10 and 15 year survival rates of $83 \%$ and $60 \%$, respectively. The study of 80 consecutive Japanese Val30Met patients (LTx, 37; untreated, 43) found similar outcomes for the untreated cohort ${ }^{37}$. Untreated patients had a mOS of 10 years (10-year survival rate of 56\%), and LTx-treated patients had prolonged survival compared with untreated patients. Notably, this study classified deaths as either related or unrelated to progressive amyloidosis and reported an outstanding $100 \%$ survival rate at 10 years among LTx treated patients $(\mathrm{n}=37)$. The different approach followed on this study on how to analyze deaths can lead to completely different survival results. The reason being because LTx per se (not related to progressive amyloidosis) is associated with an early excess mortality. The Portuguese study evaluated all-cause mortality and reported a $14 \%$ mortality rate in the first year following LTx, similar to what was found in the Swedish LTx cohort $(n=108)$ and to what has been reported using data from the Familial Amyloidosis Polyneuropathy World Transplant Registry (FAPWTR) ${ }^{17}$. The FAPWTR retrospectively reported a noteworthy improvement in overall survival among LTx cohort (20-year survival rate of 55.3\%) as compared with studies on the natural history of the disease ${ }^{38-40}$. In this study, higher modified body mass index, early onset of disease $(<50$ years of age), shorter disease duration, and TTR Val30Met rather than non-TTR Val30Met mutations in patients were independent favourable, significant factors for survival.

Some variants such as Thr60Ala and Ile107Val are associated with a more rapid and severe disease progression ${ }^{41}$ as compared to Val30Met patients, and have a shorter median survival ${ }^{42}$.

\section{Conclusion}

Observational studies are of exceptional usefulness in the context of rare diseases such as TTR-FAP because they allow investigating questions unaddressed by randomized clinical trials and are of key importance for health policy and medical decision making. Our review has found that both LTx and tafamidis are associated with statistically significant and clinically relevant survival gains. Notably, tafamidis is associated with higher patient survival rates than LTx; although the follow-up has been relatively short (as compared with LTx follow-up) for definitive conclusions to be drawn. As anticipated due to their novelty, no evidence was found for survival among patients treated with inotersen or patisiran. Altogether, this evidence shows that the prognosis for patients with TTRFAP has changed dramatically in the past 25 years, from a progressive, devastating, fatal disease to a more chronic condition with treatment leading to delayed disease progression and life expectancy gains. Improvements may be made in the prognosis of patients being timely diagnosis and early treatment initiation key aspects. However, not all patients are eligible for LTx or tafamidis treatment. Recent outstanding advances have been made in therapeutic treatment options which are much less invasive than LTx and appear to provide important benefits for patients. Longer follow-up data may facilitate understanding of how clinical practice can reach optimal treatment choice and sequence to maximize survival, while preserving the patient's health-related quality of life.

\section{Conflicts of Interest}

Mónica Inês is $\mathrm{PhD}$ candidate and Outcomes \& Evidence full-time employee of Pfizer in Portugal and hold stock and/or stock options.

\section{References}

1. Andrade C. A peculiar form of peripheral neuropathy; familiar atypical generalized amyloidosis with special involvement of the peripheral nerves. Brain. 1952; 75(3): 408-27.

2. Ando Y, Coelho T, Berk JL, et al. Guideline of transthyretin-related hereditary amyloidosis for clinicians. Orphanet J Rare Dis. 2013; 8: 31.

3. Sekijima Y. Transthyretin (ATTR) amyloidosis: clinical spectrum, molecular pathogenesis and disease-modifying treatments. J Neurol Neurosurg Psychiatry. 2015; 86(9): 1036-43.

4. Rowczenio DM, Noor I, Gillmore JD, et al. Online registry for mutations in hereditary amyloidosis including nomenclature recommendations. Hum Mutat. 2014; 35(9): E2403-12.

5. Conceicao I, Gonzalez-Duarte A, Obici L, et al. "Red-flag" symptom 
clusters in transthyretin familial amyloid polyneuropathy. J Peripher Nerv Syst. 2016; 21(1): 5-9.

6. Sekijima YYK, Tokuda T, et al. Familial Transthyretin Amyloidosis. 1993-2016. In: GeneReviews at GeneTests: Medical Genetics Information Resource [Internet]. Seattle: Seattle (WA): University of Washington. Available from: http://www.ncbi.nlm.nih.gov/books/ NBK1194.

7. Parman Y, Adams D, Obici L, et al. Sixty years of transthyretin familial amyloid polyneuropathy (TTR-FAP) in Europe: where are we now? A European network approach to defining the epidemiology and management patterns for TTR-FAP. Curr Opin Neurol. 2016; 29 Suppl 1: S3-S13.

8. Schmidt HH, Waddington-Cruz M, Botteman MF, et al. Estimating the global prevalence of transthyretin familial amyloid polyneuropathy. Muscle Nerve. 2018; 57(5): 829-37.

9. Ines M, Coelho T, Conceicao I, et al. Epidemiology of Transthyretin Familial Amyloid Polyneuropathy in Portugal: A Nationwide Study. Neuroepidemiology. 2018; 51(3-4): 177-82.

10. Sousa A, Coelho T, Barros J, et al. Genetic epidemiology of familial amyloidotic polyneuropathy (FAP)-type I in Povoa do Varzim and Vila do Conde (north of Portugal). Am J Med Genet. 1995; 60(6): 512-21.

11. Hund E. Familial amyloidotic polyneuropathy: current and emerging treatment options for transthyretin-mediated amyloidosis. Appl Clin Genet. 2012; 5: 37-41.

12. Stewart M, Shaffer S, Murphy B, et al. Characterizing the High Disease Burden of Transthyretin Amyloidosis for Patients and Caregivers. Neurol Ther. 2018

13. Holmgren G, Steen L, Ekstedt J, et al. Biochemical effect of liver transplantation in two Swedish patients with familial amyloidotic polyneuropathy (FAP-met30). Clin Genet. 1991; 40(3): 242-6.

14. Holmgren G, Ericzon BG, Groth CG, et al. Clinical improvement and amyloid regression after liver transplantation in hereditary transthyretin amyloidosis. Lancet. 1993; 341(8853): 1113-6.

15. Benson MD. Liver transplantation and transthyretin amyloidosis Muscle Nerve. 2013; 47(2): 157-62.

16. Carvalho A, Rocha A, Lobato L. Liver transplantation in transthyretin amyloidosis: issues and challenges. Liver Transpl. 2015; 21(3): 282-92.

17. Ericzon BG, Wilczek HE, Larsson M, et al. Liver Transplantation for Hereditary Transthyretin Amyloidosis: After 20 Years Still the Best Therapeutic Alternative. Transplantation. 2015; 99(9): 1847-54.

18. Telles-Correia D, Cortez-Pinto H, Barbosa A, et al. Quality of life following liver transplantation: a comparative study between Familial Amyloid Neuropathy and liver disease patients. BMC Gastroenterol. 2009; 9: 54.

19. Lopes A, Sousa A, Carvalho-Frade I, et al. Quality of life and psychosocial aspects in liver transplantation: a longitudinal survey. Transpl Int. 2015; 28(Suppl 4): 714.

20. Vollmar J, Schmid JC, Hoppe-Lotichius M, et al. Progression of transthyretin (TTR) amyloidosis in donors and recipients after domino liver transplantation-a prospective single-center cohort study. Transpl Int. 2018; 31(11): 1207-15.

21. Berk JL, Suhr OB, Obici L, et al. Repurposing diflunisal for familial amyloid polyneuropathy: a randomized clinical trial. JAMA. 2013; 310(24): 2658-67.

22. Plante-Bordeneuve V. Transthyretin familial amyloid polyneuropathy: an update. J Neurol. 2018; 265(4): 976-83.

23. Coelho T, Maia LF, Martins da Silva A, et al. Tafamidis for transthyretin familial amyloid polyneuropathy: a randomized, controlled trial. Neurology. 2012; 79(8): 785-92.

24. Coelho T, Maia LF, da Silva AM, et al. Long-term effects of tafamidis for the treatment of transthyretin familial amyloid polyneuropathy. Neurol. 2013; 260(11): 2802-14.

25. Adams D, Suhr OB, Hund E, et al. First European consensus for diagnosis, management, and treatment of transthyretin familial amyloid polyneuropathy. Curr Opin Neurol. 2016; 29 Suppl 1: S14-26.

26. Waddington Cruz M, Amass L, Keohane D, et al. Early intervention with tafamidis provides long-term (5.5-year) delay of neurologic progression in transthyretin hereditary amyloid polyneuropathy. Amyloid. 2016: 1-6.

27. Barroso FA, Judge DP, Ebede B, et al. Long-term safety and efficacy of tafamidis for the treatment of hereditary transthyretin amyloid polyneuropathy: results up to 6 years. Amyloid. 2017; 24(3): 194-204.

28. Mundayat R, Stewart M, Alvir J, et al. Positive Effectiveness of Tafamidis in Delaying Disease Progression in Transthyretin Familial Amyloid Polyneuropathy up to 2 Years: An Analysis from the Transthyretin Amyloidosis Outcomes Survey (THAOS). Neurol Ther. 2018.

29. Keohane D, Schwartz J, Gundapaneni B, et al. Tafamidis delays disease progression in patients with early stage transthyretin familial amyloid polyneuropathy: additional supportive analyses from the pivotal trial. Amyloid. 2017; 24(1): 30-6.

30. Conceicao I, Miranda B, Castro J, et al. Hereditary amyloidosis related to transthyretin V30M: disease progression in treated and untreated patients. Eur J Neurol. 2018; 25(11): 1320-e115.

31. Benson MD, Waddington-Cruz M, Berk JL, et al. Inotersen Treatment for Patients with Hereditary Transthyretin Amyloidosis. N Engl J Med. 2018; 379(1): 22-31.

32. Adams D, Gonzalez-Duarte A, O'Riordan WD, et al. Patisiran, an RNAi Therapeutic, for Hereditary Transthyretin Amyloidosis. N Engl J Med. 2018; 379(1): 11-21

33. Kristen AV, Ajroud-Driss S, Conceicao I, et al. Patisiran, an RNAi therapeutic for the treatment of hereditary transthyretin-mediated amyloidosis. Neurodegener Dis Manag. 2018.

34. Coelho T, Inês M, Conceicao I, et al. Natural history and survival in stage 1 Val30Met transthyretin familial amyloid polyneuropathy. Neurology. 2018.

35. Said G, Grippon S, Kirkpatrick P. Tafamidis. Nat Rev Drug Discov. 2012; 11(3): 185-6.

36. Okamoto S, Wixner J, Obayashi K, et al. Liver transplantation for familial amyloidotic polyneuropathy: impact on Swedish patients' survival. Liver Transpl. 2009; 15(10): 1229-35.

37. Yamashita T, Ando Y, Okamoto $\mathrm{S}$, et al. Long-term survival after liver transplantation in patients with familial amyloid polyneuropathy. Neurology. 2012; 78(9): 637-43.

38. Sousa A, Andersson R, Drugge U, et al. Familial amyloidotic polyneuropathy in Sweden: geographical distribution, age of onset, and prevalence. Hum Hered. 1993; 43(5): 288-94.

39. Coutinho P, da Silva AM, Lima JL, et al. Forty years of experience with type I amyloid neuropathy. Review of 483 cases. In: Glenner GG CP, de Freitas F, editor. Amyloid and Amyloidosis. Amsterdam Excerpta Medica. 1980; 88-98.

40. Suhr 0, Danielsson A, Holmgren G, et al. Malnutrition and gastrointestinal dysfunction as prognostic factors for survival in familial amyloidotic polyneuropathy. J Intern Med. 1994; 235(5): $479-85$

41. Carr AS, Pelayo-Negro AL, Evans MR, et al. A study of the neuropathy associated with transthyretin amyloidosis (ATTR) in the UK. J Neurol Neurosurg Psychiatry. 2016; 87(6): 620-7.

42. Mariani LL, Lozeron P, Theaudin M, et al. Genotype-phenotype correlation and course of transthyretin familial amyloid polyneuropathies in France. Ann Neurol. 2015; 78(6): 901-16. 